\title{
FEM Analysis Applied to OT Bridge Abutment with Seeger Retention System
}

\author{
Gabriele Cervino ${ }^{1}$ Marco Cicciù ${ }^{1}$ Simone Fedi ${ }^{1}$ Dario Milone ${ }^{2}$ Luca Fiorillo ${ }^{1}$
}

\author{
${ }^{1}$ Department of Biomedical and Dental Sciences and Morphological \\ and Functional Imaging, Messina University, Messina, Italy \\ 2Department of Engineering, Messina University, Messina, Italy
}

\begin{abstract}
Address for correspondence Marco Cicciù, DDS, PhD, MSc, Department of Biomedical and Dental Sciences and Morphological and Functional Imaging, Messina University, Messina 98100, ME, Italy (e-mail: mcicciu@unime.it).
\end{abstract}

\begin{abstract}
Keywords

- all-on-four

- implant

- screw

- prosthesis

- implant-abutment connections

- stress distribution

- FEM

- von Mises

Objective The purpose of this investigation is to highlight the technical components of a new kind of screw-retained dental implant prosthesis. The hypothesis is whether the OT Bridge (Rhein 83 S.R.L.; Bologna, Italy) system could be applied without secondary screw in the "all-on-four" retention system, thanks to the presence of an internal seeger. Materials and Methods By using engineering device such as finite element method (FEM) and von Mises investigation, it has been studied how the fixed prosthodontics for full-arch retention can be influenced by the presence of the screw for stabilizing it. Results In a dental implant, one model with four different configurations of the fullarch prosthesis retainer and the seeger has been investigated and then examined in contrast with or without the passant screw for locking the system. The experiments of this virtual study highlighted different features and mechanical behaviors of prosthodontic attachments.

Conclusion The first two configurations, respectively those in which there are four and three connection screws, are safe and predictable. Therefore, the presence of the seeger significantly improves the stability and the retention of the whole prosthesis.
\end{abstract}

\section{Introduction}

The clinical conditions of edentulous jaws are nowadays considered a common problem for patients and clinicians. The choice of the correct treatment plan, and its significance for the patients' quality of life, still remains a challenge due to the different ridge atrophic shapes and to the several prosthodontic solutions. The partially or totally edentulous patient experiences a condition of strong physical and social discomfort. The loss of teeth has unfavorable impact on the oral esthetics and function, involving also the quality of life of the patients. ${ }^{1-3}$ In the past 20 years, the advent of the dental implant-based therapies quickly developed as safe and predictable remedy for fully edentulous patients and as an alternative to standard removable dentures. Several treatments can include prosthodontic rehabilitation involving high or low numbers of dental implants, cemented or screw-retained dental prosthesis, and dental

DOI https://doi.org/

10.1055/s-0040-1715550

ISSN 1305-7456. implant-supported overdentures. Usually, the treatment choice is related to the patient's desires but at the same time it is chosen considering the patients anatomical conditions. Many patients, especially the oldest ones affected by severely atrophic ridges, feel uncomfortable with the removal of prosthesis, especially of the lower jaw. ${ }^{4}$ To overcome these clinical adverse conditions, two or four dental implants for full-arch prosthesis rehabilitation could be recommended as first treatment option. In this way, noninvasive surgery could be guaranteed with excellent functional and esthetic long-term clinical results, enabling recovery of patients' quality of life. The old overdenture system can be defined as a removable dental prosthesis positioned over one or more remaining natural teeth, the residual roots, and/or dental implants. The dental implant-abutment connections have to be solid and secure, considering unfavorable inclination due to the typical anatomical condition of

This is an open access article published by Thieme under the terms of the Creative Commons Attribution-NonDerivative-NonCommercial-License, permitting copying and reproduction so long as the original work is given appropriate credit. Contents may not be used for commercial purposes, or adapted, remixed, transformed or built upon. (https://creativecommons.org/licenses/by-nc-nd/4.0/)

Thieme Medical and Scientific Publishers Pvt. Ltd., A-12, 2nd Floor, Sector 2, Noida-201301 UP, India 
the atrophic maxilla. ${ }^{4}$ The present study reported a new way of stabilization of full-arch fixed prosthesis, developed by moving a step further over the old concept of overdenture.

Today, the dental prosthesis could be classified as fixed with cemented or screw-retained abutments. However, when it comes to determining the different holding systems available in terms of favorable clinical outcomes, ease of maintenance, patient satisfaction and preferences, cost to the patient, or ease of cleaning and removal, available literature still does not provide reliable data as to which is the ideal retention system for this kind of dental rehabilitation. When placed on the abutments, the prostheses, fixed or not, have to guarantee esthetics and function. This last parameter is most difficult to be achieved because the forces involved in the masticatory cycle are not linear and not homogenous. ${ }^{5,6}$ Recently, several published papers investigated the stress over the bone tissue and over dental implants and prosthodontic elements during the chewing alternations, and aimed to study the point of fracture as well as the wear of the material used.7 The uniform dispersion of the stresses that occurred on prosthodontic components during the chewing cycles is documented as being conditioned by the number and the position of the dental implants, as well as by the basic material and by the singular prosthodontic aspect and elements. One of the most common clinical problem associated with implant-supported restoration is screw loosening and then loss of retention over time. ${ }^{8}$ The purpose of the presented research is to evaluate the prosthetic elements of retention for full-arch fixed prosthesis placed over four dental implants by using OT Equator (Rhein 83 S.R.L.; Bologna, Italy) attachments and OT Bridge (Rhein 83 S.R.L.) components and the seeger to highlight potential failures related to any fracture of the structural components or any overload on bone tissue, as well as to evaluate the effectiveness of the seeger as an extra element of retention.

Therefore, the principal aim is to consider the grade of retention by using the passant screws, screwed to all or to OT Equator attachments, that are tightened into the dental implant (Osstem TSIII; Osstem Global Co., Ltd., Seoul, South Korea) according to the all-on-four technique by using finite element analysis (FEA). A comparative analysis has then been performed to finalize the systems with a type of implant (Osstem TSIII) and "abutments" connected by metric threading. ${ }^{9-11}$ Furthermore, in consequence of the absence of many parameters, pictures have been utilized to recreate the files. Potential approximations have been considered due to this operation. ${ }^{12}$ The results of the tests were recorded as graphic and data, which were matched to identify which was better among the ones studied. Finally, the von Mises stress test has been set and evaluated.

\section{Materials and Methods}

The principal factors that regulate the precision of the FEA method have been detected. In association with these, the detailed shape of the system and the peri-implant bone anatomy to be shaped, the perimeter conditions and constraints, the material mechanical features, the load conditions "repeated on time related to masticatory cycle," the bone-implant contact, the test of convergence, and the validation of the model have been involved in this analysis.

Solid representation of the jaws, dental implants, and prosthodontic elements were recreated from Roster images, which are processed through a three-dimensional computer-aided design (3D CAD) in finite element method (FEM). The search process was then separated into the following two moments: the preprocessing-finite element model's building step-and the postprocessing-converting and modeling of solutions. ${ }^{9,13}$ The following tests incorporated the tests about the dental prosthesis marked "Rhein 83 S.R.L." with the following configuration:

1. Four OT Equator-OT Bridge assembled with four passant retaining screws: Test 1

2. Four OT Equator-OT Bridge assembled with three out of four passant retaining screws: Test 2

3. Four OT Equator-OT Bridge assembled with two out of four passant retaining screws: Test 3

4. Four OT Equator-OT Bridge assembled without retaining screws: Test 4

This type of retention system (OT Equator) was born as an evolution of the spherical system. The component to be highlighted is also represented by the presence of the seeger. The subequatorial component allows the housing inside the cylindrical abutment of an interchangeable acetal ring, the seeger, and represents a systematic alternative to screwed and cemented solutions.

The threaded hole with a closed bottom on the attachment does not communicate with the implant, avoiding coronal-apical bacterial infiltrations. Thanks to this, there are also great advantages in overcoming implant disparallelisms, even in extreme cases of over $80^{\circ}$, without resorting to components such as multiunit abutment or drilling techniques. The real revolution offered by the OT Equator is versatility with the use of the same identical abutment, both for the removable and fixed prosthesis solution (-Fig. 1).

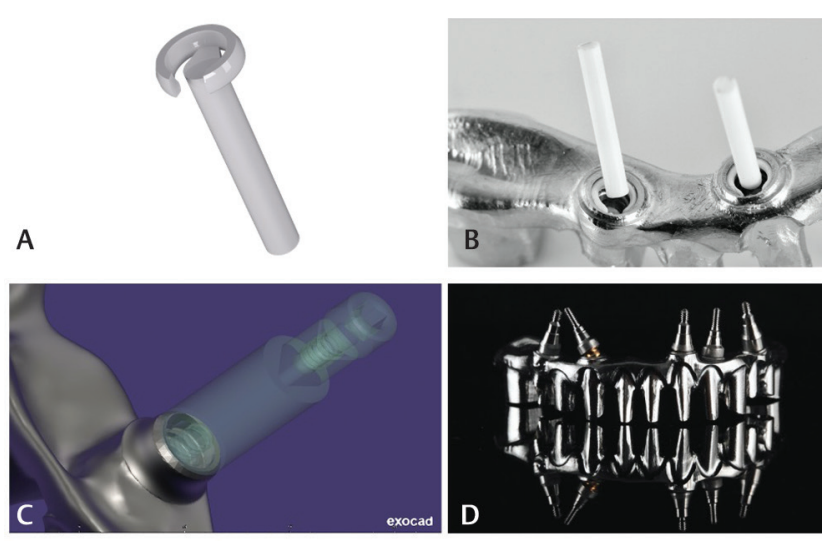

Fig. 1 OT Equator and OT bridge systems details: elastic seeger with mounter (A); elastic seeger positioning (B); exocad screenshot about screw connection (C); final prosthesis structure (D). 


\section{Reverse Engineering}

The model sizes were created from the prosthodontic elements and the pictures were made real by the small details of their physico-chemical features published in the literature and by the brand drafts. The creating moment has been developed by SolidWork, by which the information is screened from the physical system to a mathematical clone, deduced from the same number of variables and "filtering out" the delaying ones (-Fig. 2). To create a homogeneous and adequate mesh of the whole model, the discretization tests revealed the difficulty in finding optimal solutions with a small number of elements. For this reason, the mesh has been refined in areas of particular interest. The areas where there is a coupling have also been thickened, to obtain a better response along the contact interface between the components. For all the tests, the load condition considered is shown in - Table 1. To ensure that a real situation was considered, the frictions between the various components were also considered ( $\mathbf{-}$ Table $\mathbf{2}$ ).

\section{Finite Element Methods}

Therefore, after having these 3D CAD pictures, the FEA jaw-implant-prosthesis has been created by using Ansys Workbench. A 3D linear static parametric simulation has been developed underlining the ratio (stress and strain) between bone tissue and prosthodontic pieces, and fixture and OT Equator retainers.

\section{Characteristic of the Materials Involved in the Study} The identical force has been directed to the different implants and the consequent strength dispersion has been recorded. The features of the materials have been classified in terms of Young's modulus, Poisson's ratio, and density. The numerous physical characteristics of the components have been recorded regarding the occlusal and lateral strengths. The titanium alloy (Ti6Al4V) under evaluation can be classified as homogeneous, linear, and isotropic, while the bone

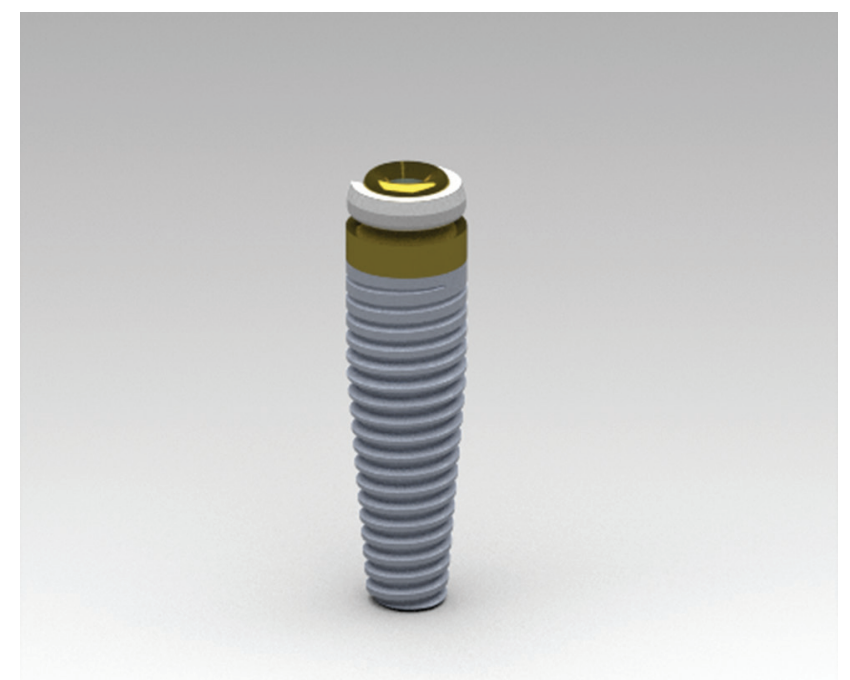

Fig. 2 Reverse engineering on a dental implant.
Table 1 Summary of the type and location of the loads applied to the system

\begin{tabular}{|l|l|}
\hline Test & Loading conditions \\
\hline Type of load & Compression load \\
\hline Location & Molars \\
\hline Condition & Osseointegration \\
\hline
\end{tabular}

Table 2 Summary of the type of contact and the numerical value used

\begin{tabular}{|l|l|l|}
\hline Connection & $\begin{array}{l}\text { Type of } \\
\text { coupling }\end{array}$ & $\begin{array}{l}\text { Coefficient } \\
\text { clutch }\end{array}$ \\
\hline Cancellous bone/cortical bone & Bonded & - \\
\hline Cortical bone/fixture & Bonded & - \\
\hline Cancellous bone/fixture & Bonded & - \\
\hline Fixture/OT Equator & Frictional & 0.2 \\
\hline OT Equator/Seeger & Frictional & 0.35 \\
\hline OT Equator/abutment & Frictional & 0.2 \\
\hline Seeger/abutment & Frictional & 0.35 \\
\hline Abutment/bridge & Bonded & - \\
\hline
\end{tabular}

tissues (cortical and cancellous) that should be anisotropic have been classified as orthotropic. Therefore, the numerous alteration parameters in the three space vectors in response to the stress have been recorded. ${ }^{13-18}$

\section{Results}

Analyzed with the other manuscripts published to date in the scientific literature, this study is presenting a reproduction as detailed as possible: interaction between the surfaces of nonpenetration with friction and preloading of the passant screw. In the past, it was common to apply a "joint" relation between the sections and not to consider preload stress, nevertheless, to the detriment of the truthfulness of the data. In this specific study, the authors obtained a balance for achieving outcomes that are as similar to reality. ${ }^{9,18-23}$

A CAD drawing of each element has been developed and then assembled in a unique model with relative adaption. At the same time, the purpose of the investigation is to research the global tension on the four groups. A compression vertical load of $800 \mathrm{~N}$ has been applied to the model.

\section{Test 1 Results}

From the analysis of the results from "Test 1" (-Figs. 3-6), it can be seen that the maximum value reached on the prosthesis is equal to $291 \mathrm{MPa}$ (tension lower than that of yield) while for the bone the value found is equal to $83 \mathrm{MPa}$. By preparing a detail of the four prostheses, removing the bone from the analysis, it can be seen that the results remain unchanged. From a preliminary analysis, it can be seen how the two posterior prostheses are more stressed than the anterior ones. The bridge is loaded, 


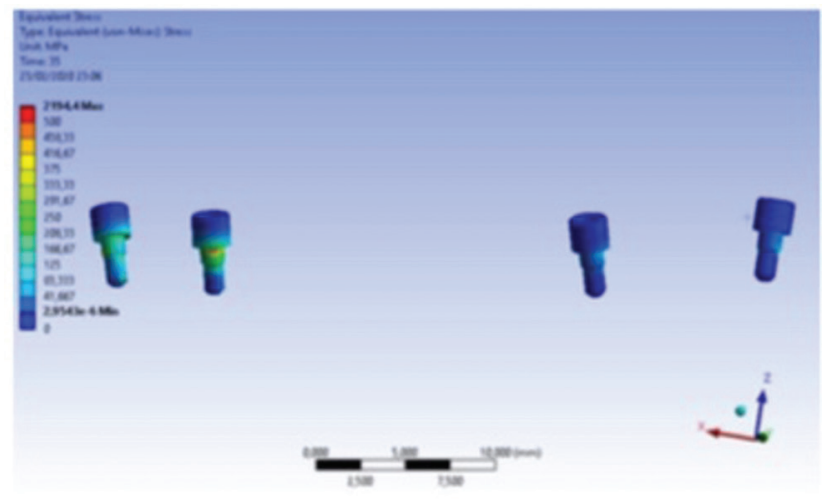

Fig. 3 Von Mises stress related to the connection screws.

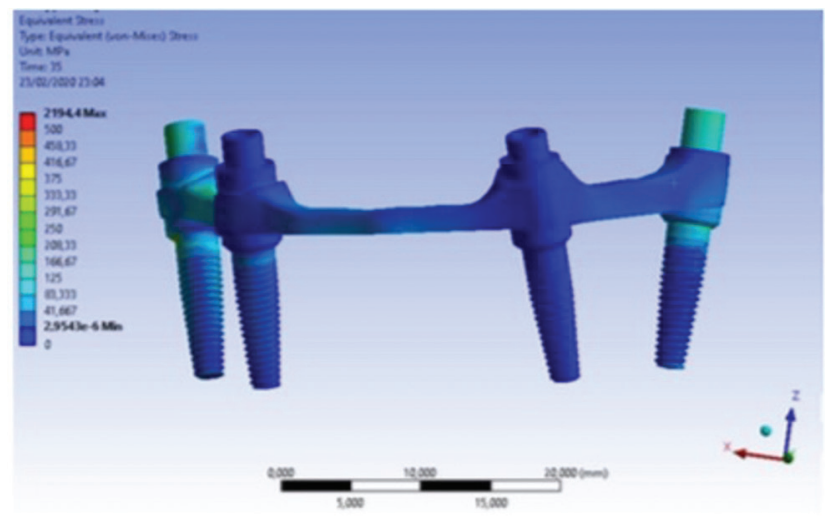

Fig. 4 Von Mises stress related to the implant.

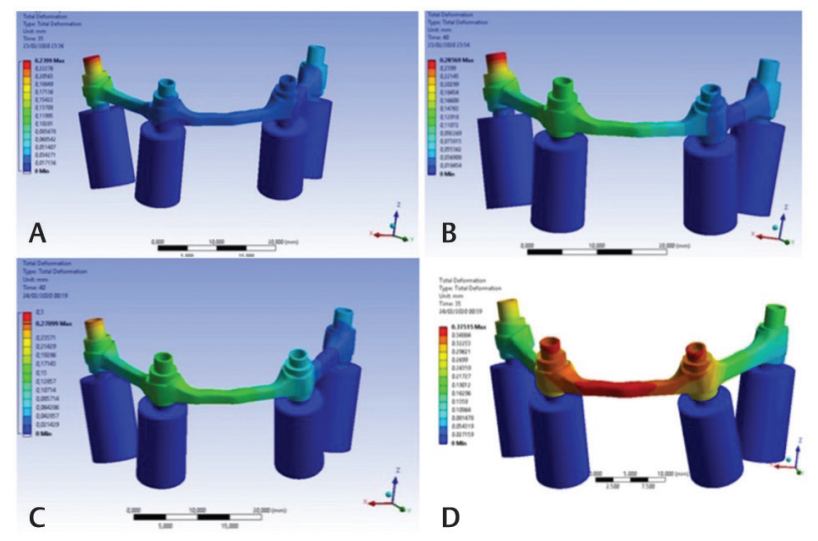

Fig. 5 Total movement of the system on Test 1(A), 2(B), 3(C), 4(D).

with greater intensity, on the left side (the prostheses are not mounted symmetrically). It can be noted that the most stressed element is the connecting screw. In particular, the most stressed screw is the left front, since the load is unloaded more on it.

For the comparison of the four system configurations, it is useful to evaluate the displacements of the system to characterize its lability. It is easy to read/note that the displacement calculated as the result of the three movements along the three axes $x, y$, and $z$ is greater in correspondence

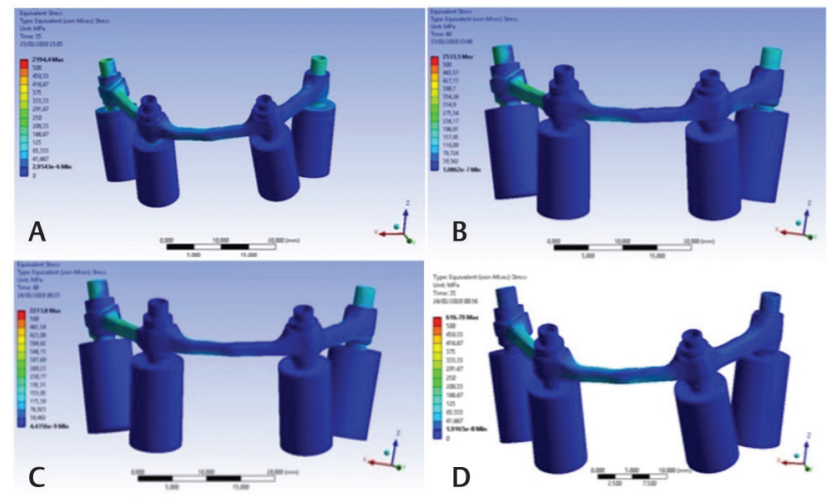

Fig. 6 Von Mises stress related to test 1(A), 2(B), 3(C), 4(D).

with the implant, on the rear left. Once the overall displacements of the system have been calculated, they are assessed along each individual axis. From the evaluation of the displacements along the $x$-axis, it can be noted that the greatest displacement along the increasing $x$ is located on the left posterior stump. Moving on to the evaluation of the displacements along the $y$-axis, a displacement can always be noticed along the positive $y$ in the same area. This points out that from the set compression load, twisting moments arise along the $x$ and $y$ axes. Ultimately, the displacements along the $z$-axis are checked, to note if there is also a bending of the implant beyond the torsion. This analysis shows that there is a displacement along the positive $z$ precisely on the anteriorly mounted prostheses.

The results obtained from "Test 1" show that the most stressed elements are the connection screws. It can also be noted that the displacement modules along the three axes are contained around $0.1 \mathrm{~mm}$. It can be concluded that the implant is fixed, and resists under a static load of $400 \mathrm{~N}$ per molar.

\section{Test 2 Results}

From the analysis of the results from "Test 2" (-Fig. 3-6), it can be seen that the maximum value reached on the prosthesis is equal to $314 \mathrm{MPa}$ (tension lower than that of yield) while for the bone the value found is equal to $118 \mathrm{MPa}$. A different distribution of the tensions can be seen, given the fact that the implants are constrained on three surfaces and no longer on four. Furthermore, the rear left screw is more loaded than in the previous case, even if the peaks are found in the front right one.

Moving on to the evaluation of the displacements along the $y$-axis, a displacement can always be noticed along the negative $y$ in the same area. This points out that from the set compression load, twisting moments arise along the $x$ and $y$ axes.

Ultimately, the displacements along the $z$-axis are checked, to note if there is also a bending of the implant beyond the torsion. From this analysis, it appears that there is a displacement along the positive $z$ precisely on the prostheses mounted anteriorly, especially in the nonanchored area.

The results obtained from "Test 2" show that the most stressed elements are the connection screws. It can also be noted that the modules of the movements along the three 
axes are contained around $0.2 \mathrm{~mm}$. It can be concluded that the implant is once again fixed, and resists under a static load of $400 \mathrm{~N}$ for molars.

\section{Test 3 Results}

From the analysis of the results from "Test 3," as shown in -Figs. 3-6, it can be seen that the maximum value reached on the prosthesis is equal to $346 \mathrm{MPa}$ (lower tension than the yield strength) while for the bone the value found is equal to $153 \mathrm{MPa}$.

By preparing a detail of the four prostheses, removing the bone from the analysis, it can be seen that the results remain unchanged. From a preliminary analysis, it can be seen how the two posterior prostheses are more stressed than the anterior ones. The bridge is loaded, with greater intensity, on the left side (the prostheses are not mounted symmetrically).

A graph was drawn relating to the trend of the voltages generated at the equator interface/connection screw, to examine how the voltage state in this area varies. It can be noted that the most stressed element is the connecting screw. In particular, the most stressed screw is the one on the rear left, since the load is more discharged on it.

A different distribution of the tensions can be seen, given the fact that the implants are constrained on two surfaces and no longer on four. Furthermore, the rear left screw is more loaded than in the previous case. Moving on to the evaluation of the displacements along the $y$-axis, a displacement can always be noticed along the negative $y$ in the same area. This points out that from the set compression load, twisting moments arise along the $x$ and $y$ axes.

Ultimately, the displacements along the $z$-axis are checked, to note if there is also a bending of the implant beyond the torsion. This analysis shows that there is a displacement along the positive $z$ precisely on the anteriorly mounted prostheses.

The results obtained from "Test 3" show that loading the system with $87 \%$ of the total load leads to a condition in which the resultant of the movements reaches a value around $0.3 \mathrm{~mm}$, a value for which it could have system instability.

\section{Test 4 Results}

From the analysis of the results from "Test 4," as shown in -Figs. 3-6, it can be seen that the maximum value reached on the prosthesis is equal to $208 \mathrm{MPa}$ (lower than the yield stress) while for the bone the value found is equal to $83 \mathrm{MPa}$.

By preparing a detail of the four prostheses, removing the bone from the analysis, it can be seen that the results remain unchanged. From a preliminary analysis, it can be seen how the two posterior implants are more stressed than the anterior ones. The bridge is loaded, with greater intensity, on the left side (the prostheses are not mounted symmetrically).

It can be noted that the most stressed element is the bridge. A different distribution of the strain can be seen. Furthermore, using this setup, it can be noted that the OT Equator attachment is more stressed than in previous cases, since as it is no longer anchored with the connection screws; the stump by rotating applies pressure on the OT Equator attachments.

For the comparison of the four system configurations, it is useful to evaluate the displacements of the system to characterize its lability. It is easy to read/note that the displacement calculated as the result of the three movements along the three axes $x, y$, and $z$ is greater in correspondence with the two front implants.

Once the overall displacements of the system have been calculated, they are assessed along each individual axis. From the evaluation of the displacements along the $x$-axis, it can be noted that the greatest displacement along the increasing $x$ is located on the left posterior stump.

Ultimately, the displacements along the $z$-axis are checked, to note if there is also a bending of the implant beyond the torsion. This analysis shows that there is a displacement along the positive $z$ precisely on the anteriorly mounted prostheses.

The results obtained from "Test 4" show that loading the system with $12 \%$ of the total load leads to a condition in which the resultant of the movements reaches a value around $0.4 \mathrm{~mm}$. It can be concluded that this system is unstable. It is therefore not advisable to use such a conformation.

Even all the developed prosthodontic elements showed a typical system associated to the chewing cycles; the most stressed component of the fixture is represented by the connecting screw. ${ }^{1,24-27}$ Bar overdenture is a common and well-adaptable treatment option because of its stress distribution, but its cost is high and patients sometimes choose to have higher results with lower expenses (-Figs. 4-6). ${ }^{15-17,28}$

\section{Discussion}

Recently, several published papers highlighted how in rehabilitative dentistry the parameters of knowledge relating to screw retention, prosthodontic properties, and the dental implant osteointegration phenomena are fundamental for having a long-term clinical success. A further analysis about the biomechanical parameters of the oral cavity anatomy and physiology is important for the knowledge of the bone mechanical properties as well as for a precise data on the jawbone shape and size..$^{29,30}$ In the past 20 years, biomaterial internal geometry and structure have largely assisted in the integration of FEM in the product realization process. ${ }^{5,31}$

The advantages of FEM in the biomedical area are several. The most actual is connected to the chance of FEM that can enable early device efficiency testing prior to costly prototyping and bench testing. ${ }^{32,33}$ For the four tests, it was chosen to apply a load of different entity. This opportunity is dictated by the fact that by eliminating one anchor at a time, it could be seen that the displacements and tensions have an increasingly accentuated variation. Paying attention to the displacements, related to the system, it can be seen that in the last test, where it was chosen to stress the system with a load that is $12 \%$ of the total, the displacement of the system is four times greater than the first test in which all the prostheses were anchored with connection screws. 
It can be noted that although in the last test it was chosen to stress the system with a load that is $12 \%$ of the total, the resulting stress is $60 \%$ compared with the maximum stress calculated in the four tests. ${ }^{34,35}$ In the field of dentistry, the shape of prosthodontic instruments for retained overdenture base and for fixing full-arch denture has been amply studied in the recent literature, for researching the integration and the wear influenced by the masticatory cycles. The OT Equator attachments have been recently studied because it is on trade just by 2007. This retainer can be applied both for overdenture with direct connection and for overdenture placed over a secondary structure. ${ }^{36} \mathrm{~A}$ review and meta-analysis performed by Keshk et $\mathrm{al}^{37}$ published on 2017 about full-arch dental fixation systems and about their conditioning over the peri-implant bone loss showed how there are no statistically significant anomalies between the kinds of overdenture attachments with regard to marginal bone loss, bleeding index, gingival index, and plaque index. ${ }^{37-44}$ The real innovation in this method of attachment lies precisely in the presence of an acetal ring, called seeger $(\boldsymbol{-}$ Fig. $\mathbf{1})$, which guarantees stability and retention to the prosthesis even in the absence of screwing systems, thus promoting a distribution of forces.

\section{Conclusion}

It could therefore be concluded that the first two configurations, respectively those in which there are four connection screws and three connection screws, are safe, since the stresses generated are lower than the yield points of the material. Test 3 , in which only two connecting screws are used, highlights the possible instability of the system. In this case, although the load is $87 \%$ compared with the loads applied in the previous tests, the tensions that arise are 1.5 times higher than the first two tests. Finally, the last test highlights, as previously discussed, the possible instability of the system due to the failure to anchor with implant connection screws. The screw methods are a guarantee of fixation; however, the presence of the seeger significantly improves the stability and the retention of the whole prosthesis, as demonstrated by the tests. Higher, long-term stability could be expected using four and three screws. The new shape and the presence of seeger could be useful for increasing the stability of a fullarch prosthesis system delivered on four implants, particularly when one out of four screws is excluded. Clinical studies are recommended to confirm those results.

\section{Conflict of Interest}

None declared.

\section{Acknowledgment}

The authors thank Prof. Alan Scott Herford (Loma Linda University) for checking English style and grammar of the whole article.

\section{References}

1 Müller J. Archiv für Anatomie. Physiologie und Wissenschaftliche Medicin 1886;1886:1
2 De Stefano R, Bruno A, Muscatello MR, Cedro C, Cervino G, Fiorillo L. Fear and anxiety managing methods during dental treatments: a systematic review of recent data. Minerva Stomatol 2019;68(6):317-331

3 De Stefano R. Psychological factors in dental patient care: odontophobia. Medicina (Kaunas) 2019;55(10):678

4 Cawood JI, Howell RA. Reconstructive preprosthetic surgery. I. Anatomical considerations. Int J Oral Maxillofac Surg 1991;20(2):75-82

5 Tallarico M, Caneva M, Baldini N, et al. Patient-centered rehabilitation of single, partial, and complete edentulism with cemented- or screw-retained fixed dental prosthesis: The First Osstem Advanced Dental Implant Research and Education Center Consensus Conference 2017. Eur J Dent 2018;12(4):617-626

6 Scrascia R, Fiorillo L, Gaita V, Secondo L, Nicita F, Cervino G. Implant-supported prosthesis for edentulous patient rehabilitation. From temporary prosthesis to definitive with a new protocol: a single case report. Prosthesis 2020;2(1):10:24

7 Fiorillo L, D'Amico C, Turkina AY, Nicita F, Amoroso G, Risitano G. Endo and exoskeleton: new technologies on composite materials. Prosthesis 2020;2(1):1-9

8 Cervino G, Fiorillo L, Arzukanyan AV, Spagnuolo G, Campagna P, Cicciù M. Application of bioengineering devices for stress evaluation in dentistry: the last 10 years FEM parametric analysis of outcomes and current trends. Minerva Stomatol 2020;69(1):55-62

9 Cervino G, Romeo U, Lauritano F, et al. Fem and Von Mises analysis of OSSTEM®dental implant structural components: evaluation of different direction dynamic loads. Open Dent J 2018;12:219-229

10 Zarone F, Apicella A, Nicolais L, Aversa R, Sorrentino R. Mandibular flexure and stress build-up in mandibular fullarch fixed prostheses supported by osseointegrated implants. Clin Oral Implants Res 2003;14(1):103-114

11 Albrektsson T, Zarb G, Worthington P, Eriksson AR. The longterm efficacy of currently used dental implants: a review and proposed criteria of success. Int J Oral Maxillofac Implants 1986;1(1):11-25

12 Gherlone E, Capparé P, Vinci R, Ferrini F, Gastaldi G, Crespi R. Conventional versus digital impressions for "all-on-four" restorations. Int J Oral Maxillofac Implants 2016;31(2):324-330

13 Cicciù M, Risitano G, Maiorana C, Franceschini G. Parametric analysis of the strength in the "Toronto" osseous-prosthesis system. Minerva Stomatol 2009;58(1-2):9-23

14 Cicciu M, Bramanti E, Matacena G, Guglielmino E, Risitano G. FEM evaluation of cemented-retained versus screw-retained dental implant single-tooth crown prosthesis. Int J Clin Exp Med 2014;7(4):817-825

15 Haack JE, Sakaguchi RL, Sun T, Coffey JP. Elongation and preload stress in dental implant abutment screws. Int J Oral Maxillofac Implants 1995;10(5):529-536

16 Versluis A, Korioth TW, Cardoso AC. Numerical analysis of a dental implant system preloaded with a washer. Int J Oral Maxillofac Implants 1999;14(3):337-341

17 van Steenberghe D, Lekholm U, Bolender C, et al. Applicability of osseointegrated oral implants in the rehabilitation of partial edentulism: a prospective multicenter study on 558 fixtures. Int J Oral Maxillofac Implants 1990;5(3):272-281

18 Esposito M, Hirsch JM, Lekholm U, Thomsen P. Biological factors contributing to failures of osseointegrated oral implants. (II). Etiopathogenesis. Eur J Oral Sci 1998;106(3):721-764

19 Cicciù M, Cervino G, Milone D, Risitano G. FEM analysis of dental implant-abutment interface overdenture components and parametric evaluation of Equator ${ }^{\circledR}$ and Locator ${ }^{\circledR}$ prosthodontics attachments. Materials (Basel) 2019;12(4):59210.3390/ ma12040592 
20 Cicciù M, Cervino G, Milone D, Risitano G. FEM investigation of the stress distribution over mandibular bone due to screwed overdenture positioned on dental implants. Materials (Basel) 2018;11(9):1512

21 Cicciù M, Cervino G, Bramanti E, et al. FEM analysis of mandibular prosthetic overdenture supported by dental implants: evaluation of different retention methods. Comput Math Methods Med 2015;2015:943839

22 Cicciù M, Bramanti E, Cecchetti F, Scappaticci L, Guglielmino E, Risitano G. FEM and Von Mises analyses of different dental implant shapes for masticatory loading distribution. Oral Implantol (Rome) 2014;7(1):1-10

23 Bramanti E, Cervino G, Lauritano F, et al. FEM and Von Mises analysis on prosthetic crowns structural elements: evaluation of different applied materials. ScientificWorldJournal 2017;20 17:102957410.1155/2017/1029574

24 DE Vico G, Bonino M, Spinelli D, et al. Rationale for tilted implants: FEA considerations and clinical reports. Oral Implantol (Rome) 2011;4(3-4):23-33

25 Di Salle A, Spagnuolo G, Conte R, Procino A, Peluso G, Rengo C. Effects of various prophylactic procedures on titanium surfaces and biofilm formation. J Periodontal Implant Sci 2018;48(6):373-382

26 Vayron R, Nguyen V-H, Lecuelle B, et al. Comparison of resonance frequency analysis and of quantitative ultrasound to assess dental implant osseointegration. Sensors (Basel) 2018;18(5):139710.3390/s18051397

27 Tallarico M, Baldini N, Martinolli M, et al. Do the new hydrophilic surface have any influence on early success rate and implant stability during osseointegration period? Four-month preliminary results from a split-mouth, randomized controlled trial. Eur J Dent 2019;13(1):95-101

28 Mailath G, Stoiber B, Watzek G, Matejka M. [Bone resorption at the entry of osseointegrated implants-a biomechanical phenomenon. Finite element study]. Z Stomatol 1989;86(4):207-216 Die Knochenresorption an der Eintrittstelle osseointegrierter Implantate-ein biomechanisches Phanomen. Eine Finite-Element-Studie.

29 Meijer HJ, Starmans FJ, Steen WH, Bosman F. Location of implants in the interforaminal region of the mandible and the consequences for the design of the superstructure. J Oral Rehabil 1994;21(1):47-56

30 Meijer HJ, Kuiper JH, Starmans FJ, Bosman F. Stress distribution around dental implants: influence of superstructure, length of implants, and height of mandible. J Prosthet Dent 1992;68(1):96-102

31 Clift SE, Fisher J, Watson CJ. Finite element stress and strain analysis of the bone surrounding a dental implant: effect of variations in bone modulus. Proc Inst Mech Eng $\mathrm{H}$ 1992;206(4):233-241

32 Lakes RS, Katz JL, Sternstein SS. Viscoelastic properties of wet cortical bone-I. Torsional and biaxial studies. J Biomech 1979;12(9):657-678
33 Brown CU, Norman TL, Kish VL III, Gruen TA, Blaha JD. Timedependent circumferential deformation of cortical bone upon internal radial loading. J Biomech Eng 2002;124(4):456-461

34 Alikhasi M, Rohanian A, Ghodsi S, Kolde AM. Digital versus conventional techniques for pattern fabrication of implant-supported frameworks. Eur J Dent 2018;12(1):71-76

35 Eraslan O, Inan O, Secilmis A. The effect of framework design on stress distribution in implant-supported FPDs: a 3-D FEM study. Eur J Dent 2010;4(4):374-382

36 Takeshita S, Kanazawa M, Minakuchi S. Stress analysis of mandibular two-implant overdenture with different attachment systems. Dent Mater J 2011;30(6):928-934

37 Keshk AM, Alqutaibi AY, Algabri RS, Swedan MS, Kaddah A. Prosthodontic maintenance and peri-implant tissue conditions for telescopic attachment-retained mandibular implant overdenture: systematic review and meta-analysis of randomized clinical trials. Eur J Dent 2017;11(4):559-568

38 Herford AS, Cooper TC, Maiorana C, Cicciù M. Vascularized connective tissue flap for bone graft coverage. J Oral Implantol 2011;37(2):279-285

39 Iovino P, Di Sarno A, De Caro V, Mazzei C, Santonicola A, Bruno V. Screwdriver aspiration during oral procedures: a lesson for dentists and gastroenterologists. Prosthesis 2019;1(1):61-68

40 Meloni SM, Tallarico M, Pisano M, Xhanari E, Canullo L. Immediate loading of fixed complete denture prosthesis supported by 4-8 implants placed using guided surgery: a 5-year prospective study on 66 patients with 356 implants. Clin Implant Dent Relat Res 2016;37(5):610-621

41 Tallarico M, Meloni SM. Retrospective analysis on survival rate, template-related complications, and prevalence of peri-implantitis of 694 anodized implants placed using computer-guided surgery: results between 1 and 10 years of follow-up. Int J Oral Maxillofac Implants 2017;32(5):1162-1171

42 Pozzi A, Tallarico M, Mangani F, Barlattani A. Different implant impression techniques for edentulous patients treated with CAD/CAM complete-arch prostheses: a randomised controlled trial reporting data at 3 year post-loading. Eur J Oral Implantology 2013;6(4):325-340

43 Pozzi A, Tallarico M, Moy PK. Immediate loading with a novel implant featured by variable-threaded geometry, internal conical connection and platform shifting: three-year results from a prospective cohort study. Eur J Oral Implantology 2015;8(1):51-63

44 Tallarico M, Scrascia R, Annucci M, et al. Errors in implant positioning due to lack of planning: a clinical case report of new prosthetic materials and solutions. Materials (Basel) 2020;13(8):1883 